\title{
A Retrospective Evaluation of Adverse Drug Reactions Due to Cancer Chemotherapy in a Tertiary Care Hospital in South India
}

\author{
Pai Sunil Bellare', Kamath Ashwin', Saxena Prakash PU², Sayeli Vinaykumar', Rakesh KB' \\ 'Department of Pharmacology, Kasturba Medical College, Manipal University, Mangalore, Karnataka, INDIA. \\ 2Department of Radiotherapy and Oncology, Kasturba Medical College Hospital, Attavar, Manipal Univeristy, Mangalore, Karnataka, INDIA
}

\begin{abstract}
Introduction: To evaluate the adverse drug reactions (ADRs) due to cancer chemotherapy in a tertiary care hospital in South India. Materials and Methods: This was a retrospective descriptive study. The data was obtained from patients who developed, at least, one ADR due to cancer chemotherapy during the period June 2014 to May 2015. The causality of the ADRs were evaluated by WHO-UMC causality assessment system and Naranjo's ADR probability scale, the severity of ADRs were assessed by Hartwig and Siegel ADR severity assessment scale, and preventability of ADRs were determined by the Schumock and Thornton ADR preventability assessment scale. Results: One hundred fourteen ADRs due to cancer chemotherapy were reported in 73 patients. Hyponatremia (20.2\%), neutropenia (11.4\%), infections of the lung $(11.4 \%)$, leucopenia $(9.6 \%)$ were the commonly suspected ADRs. Cisplatin (62.3\%), carboplatin (18.4\%), cetuximab (3.5\%), gefitinib $(2.6 \%)$ were the commonly suspected medications. As per the WHO-UMC causality assessment system 66(57.8\%) ADRs were possible and $48(42.1 \%)$ were probable. Naranjo's ADR probability scale showed that $74(64.9 \%)$ ADRs were possible and 40 (35.1\%) were probable. There was a very good agreement between the two scales (kappa=0.853). All ADRs
\end{abstract}

were classified as level 4 severity barring one. None of the ADRs reported were preventable. Conclusion: Since $80 \%$ of the ADRs were suspected to be due to platinum containing anticancer drugs this provides a focus for further research on devising methods for prevention or early detection of ADRs. Prompt detection of ADRs is important to decrease morbidity and mortality.

Key words: Adverse Drug Reaction, Cancer Chemotherapy, Naranjo's ADR probability scale, Causality assessment, Pharmacovigilance, WHO-UMC causality assessment system.

Correspondence :

Ashwin Kamath,

Department of Pharmacology, Kasturba Medical College, Manipal University, Light House Hill Road, Mangalore, Karnataka, INDIA.

Phone no: +919844262808

E-mail: ashwin.kamath@manipal.edu

DOI: 10.5530/jyp.2016.3.14

\section{INTRODUCTION}

A number of studies have reported the incidence of adverse drug reactions (ADR) among hospitalized patients to be between $1.5 \%$ to $6.7 \% .^{1-3}$ It is one of the leading cause of morbidity and mortality with an estimated economic burden of about 30 billion to 130 billion US dollars annually. ${ }^{4}$ ADRs are an inevitable, albeit unwanted aspect of cancer chemotherapy. The US Food and drug administration has approved more than 150 new anticancer drugs in past 20 years. Cancers which were previously fatal like testicular cancer, lymphomas, and leukemia, now have a treatment. Patients with cancer following surgery, can now prevent tumor relapse and prolong their life with adjuvant chemotherapy. Multimodal approach with chemoradiation of locally advanced cancers has now allowed for a more limited surgery. ${ }^{5}$ However, the newer drugs are also associated with a significant risk of ADRs. ${ }^{6}$ This is despite the various preventive concomitant medications administered to tackle the ADRs.

The data regarding ADRs collected during the different phases of clinical trials before regulatory approval is invariably incomplete. Moreover, there are differences in the occurrence of ADRs between countries, with locally derived data having greater relevance. Many of the ADRs are preventable and due diligence is necessary to avoid drugs which are problematic and monitor drugs with predictable toxicity. So ADR monitoring becomes an important tool to detect uncommon and sometimes serious ADRs, ensuring patient safety. ${ }^{4,7}$ Hence we conducted this study to assess the adverse drug reactions due to cancer chemotherapy in a tertiary care hospital in South India.

\section{MATERIALS AND METHODS}

This was a retrospective descriptive study. The data was obtained from patients who developed, at least, one ADR due to cancer chemotherapy during the period June 2014 to May 2015 in a tertiary care hospital in South India. It was conducted after receiving approval from the Institutional Ethics Committee. All the ADRs which were reported by the Oncology department between June 2014 to May 2015 were assessed. The data was obtained from the suspected adverse drug reaction reporting form used in the hospital for reporting ADRs to the ADR monitoring center as a part of the Pharmacovigilance program of India. The causality of the ADRs were evaluated by using the WHO-UMC causality assessment system and Naranjo's ADR probability scale. The causality was assessed by two study investigators independently and any discrepancy in the causality assessed was resolved by consensus. The severity of ADRs were assessed by Hartwig and Siegel ADR severity assessment scale, and preventability of ADRs were determined by the Schumock and Thornton ADR preventability assessment scale.

The WHO-UMC causality assessment system evaluates the causality of the ADRs and categorizes them as Certain, Probable/Likely, Possible, Unlikely, Conditional/Unclassified and Un-assessable/Unclassifiable. ${ }^{8}$ The Naranjo's ADR probability scale also evaluates the causality of the ADRs but categorizes them as Definite, Probable, Possible and Doubtful. ${ }^{9}$ The Hartwig and Siegel ADR severity assessment scale assesses the severity of the ADR and classifies them from Level 1 to Level $7 .{ }^{10}$ The Schumock and Thornton ADR preventability assessment scale determines the preventability of the ADRs and labels them as Preventable and Not preventable. ${ }^{11}$

The data was analyzed using SPSS (Statistical Package for the Social Sciences), IBM Corporation, version 20 and summarized using frequencies and percentages. The measure of agreement between the WHO-UMC causality assessment system and Naranjo's ADR probability scale was done using kappa $(\mathrm{K})$ test. 
SUNIL BELLARE et al:: Adverse Drug Reaction due to Cancer Chemotherapy

Table 1: Gender wise distribution of various malignancies in the study sample

\begin{tabular}{cccccc}
\hline & Males & \multicolumn{3}{c}{ Females } \\
\hline Malignancy & Frequency (N) & Percentage (\%) & Malignancy & Frequency (N) & Percentage (\%) \\
\hline Esophagus & 17 & 27.0 & Cervix uteri & 17 & 33.3 \\
Oropharynx & 11 & 17.5 & Breast & 08 & 15.7 \\
Nasopharynx & 09 & 14.3 & Submandibular gland & 05 & 11.8 \\
Base of the tongue & 06 & 9.5 & Hypopharynx & 04 & 7.8 \\
Larynx & 04 & 6.3 & Thyroid gland & 04 & 7.8 \\
Penis & 04 & 6.3 & Base of the tongue & 04 & 7.8 \\
Hypopharynx & 03 & Cheek mucosa & 02 & 3.9 \\
Others & 09 & 14.3 & Others & 06 & 11.9 \\
Total & 63 & 100 & Total & 51 & 100 \\
\hline
\end{tabular}

\begin{tabular}{ccc} 
Tables 2: Distribution of suspected adverse drug reactions due cancer chemotherapy \\
\hline Suspected Adverse Drug Reaction & Frequency (N) & Percentage (\%) \\
\hline Hyponatremia & 23 & 20.2 \\
Neutropenia & 13 & 11.4 \\
Infection - Lung & 13 & 11.4 \\
Leucopenia & 11 & 9.6 \\
Anemia & 11 & 9.6 \\
Thrombocytopenia & 10 & 8.8 \\
Hypokalemia & 07 & 6.1 \\
Diarrhea & 06 & 5.3 \\
Fever & 05 & 4.4 \\
Renal Failure & 05 & 4.4 \\
Others & 10 & 9 \\
\hline
\end{tabular}

Table 3: Distribution of suspected medication causing adverse drug reaction

\begin{tabular}{ccc}
\hline Suspected Medication & Frequency $(\mathrm{N})$ & Percentage (\%) \\
\hline Cisplatin & 71 & 62.3 \\
Carboplatin & 21 & 18.4 \\
Cetuximab & 4 & 3.5 \\
Gefitinib & 3 & 2.6 \\
Sunitinib & 3 & 2.6 \\
Paclitaxel & 3 & 2.6 \\
Temozolamide & 2 & 1.8 \\
Cytarabine & 2 & 1.8 \\
Others & 5 & 4.5 \\
\hline
\end{tabular}

\section{RESULTS}

One hundred fourteen ADRs due to cancer chemotherapy were reported in 73 patients during the study period. Among the ADRs reported, $63(55.3 \%)$ were in males and 51(44.7\%) in females. The mean age of the patients was $55.98 \pm 6.53$ years in males and $52.96 \pm 10.09$ years in females. Oropharyngeal malignancy $(24.6 \%)$ was the most common groups of cancers followed by malignancy affecting the digestive organs (16.7\%), female genital organs (15.8\%) and breast (7\%). Among these, malignancy of cervix uteri (14.9\%) and esophagus (14.9\%) were the most common cancers. The gender-wise distribution of different malignancies is shown in Table 1.

ADRs affecting blood (38.5\%) were the most common suspected category of ADR, followed by metabolic abnormalities (29.8\%) and infections (15\%). Table 2 shows the various ADRs reported in the study population. Hyponatremia (12\%), respiratory tract infection (9\%) and neutropenia (7\%) accounted for $44.4 \%$ of the ADRs in males. Hyponatremia (21.6\%); neutropenia, diarrhea, anemia (11.8\%); leucopenia, thrombocytopenia (9.8\%) accounted for $76.6 \%$ of the ADRs in females. Seventy-four (64.9\%) ADRs were considered to be recovering at the time of reporting, 
Table 4: Causality assessment using WHO-UMC causality assessment system and Naranjo's adverse drug reaction probability scale

\begin{tabular}{ccccc}
\hline \multirow{2}{*}{ Causality assessment } & \multicolumn{2}{c}{ Naranjo's adverse drug reaction probability } & Scale & \multirow{2}{*}{ Total } \\
\cline { 3 - 4 } & & Probable & Possible & \\
\hline \multirow{2}{*}{$\begin{array}{c}\text { WHO-UMC causality } \\
\text { assessment system }\end{array}$} & Probable & 40 & 08 & 48 \\
Total & Possible & 0 & 66 & 66 \\
\hline
\end{tabular}

$28(24.6 \%)$ were considered to have recovered $11(9.6 \%)$ were continuing and $1(0.9 \%)$ was fatal.

The most common suspected class of medicateons were platinum compounds $(81.6 \%)$ followed by tyrosine kinase inhibitors (5.6\%). Cisplatin (62.3\%) was the most common suspected medication followed by carboplatin (18.4\%) (Table 3).

As per the WHO-UMC causality assessment system 66 (57.8\%) ADRs were possible and $48(42.1 \%)$ were probable. Naranjo's ADR probability scale showed that 74 (64.9\%) ADRs were possible and 40 (35.1\%) were probable (Table 4). There was avery good agreement between the two scales of assessment (kappa $=0.853$ ). The Hartwig and Siegel ADR severity assessment scale classified all the ADRs as level 4 severity barring one, which was level 7. None of the ADRs reported were preventable as per the Schumock and Thornton ADR preventability assessment scale.

\section{DISCUSSION}

In our study, we evaluated the ADRs due to cancer chemotherapy in a tertiary care hospital in South India. The mean age of the patients was $54.63 \pm 8.40$ years. There is a link between increasing age and incidence of ADRs. This may be due to change in the pharmacokinetics and pharmacodynamics of the drug with increasing age. In addition to this, chronic illness, treatment with multiple medications also add to the burden of ADRs. ${ }^{12-14}$ Among the ADRs reported, 55.3\% were in males and $44.7 \%$ in females. A review of 48 cohort studies in the UK showed that females are more commonly affected than males, which was attributed to increased consulting rates for women compared to men in these studies. ${ }^{14}$ As a group, oropharyngeal cancers were the commonest cancer reported in our study, although individually malignancy of the cervix was the commonest cancer followed by malignancy of the esophagus. Surendiran et al. reported cervical cancer as the commonest cancer. ${ }^{15}$ While, Mallik et al and Poddar et al have reported lung and breast cancer as common cancers. ${ }^{16,17}$ The incidence and type of cancer affecting individuals show a geographical variation, which may have been responsible for this difference noted between the studies. ${ }^{18}$

In general, ADRs affecting the bloodwere the most common suspected category of ADR, Individually, hyponatremia was the commonest suspectted ADR followed by neutropenia and lung infections. All ADRs reported in our study were of level 4 severity, barring one which was fatal. Mallik et al. reported neutropenia as the commonest suspected ADR followed by emesis and alopecia. ${ }^{16}$ While, Poddar et al. reported elevated ESR, alopecia, vomiting and neutropenia as the common suspected ADRs. ${ }^{17}$ And Surendiran et al. reported nausea, alopecia, anorexia and vomiting as the suspected common ADRs. ${ }^{15}$ Except neutropenia, other ADRs were not reported in our study. A possible reason could be that the oncologists reported only those ADRs which they deemed significant and/or severe.

Proximal convoluted tubules, an important site of sodium and water reabsorption in the kidney is damaged by chemotherapeutic agents leading to hyponatremia. ${ }^{19}$ These agents also suppress the hematopoietic system, impairing host protective mechanisms leading to cytopenia's like neutropenia, anemia, and thrombocytopenia. Neutrophils are an important component of innate immunity and form the first line of defense against exogenous infections. Neutropenia caused by these agents blunts the immune response predisposing patients to infections. ${ }^{20}$ Platinum compounds like cisplatin and carboplatin were the commonlysuspected medication. These findings are similar to a previous study reported by Mallik et al. ${ }^{16}$

None of the ADRs reported in our study were assessed to be preventable. Surendiran et al. reported that ADRs like nausea and vomiting were definitely preventable. This difference may have been due to ADRs like nausea and vomiting not being reported in our study.

The causality assessent by both the WHO-UMC causality assessment system and Naranjo's ADR probability scale showed that most of the ADRs were possible. Previous studies have all shown that most common causality was 'possible. ${ }^{15-17}$ There was a very good agreement between the two scales of assessment. While, Mittal et al. had reported good agreement between the scales, Machedo et al. reported moderate agreement between the scales and Behelkar et al. reported poor agreement between the scales. ${ }^{21-23}$ Studies have shown that causality assessment of ADR is subjective, imprecise and low level of agreement exists between two observers. ${ }^{24,25}$ This may be responsible for the difference noted between the studies. We tried to ensure objective evaluation of causality by two investigators independently assessing the ADRs.

The main limitations of the study are that it was a retrospective study and included only ADRs which were spontaneously reported by the oncologist. Incomplete documentation of ADRs, particularly the description of the adverse reaction might have led to the incorrect assessment of causality and preventability by Schumock and Thornton ADR preventability assessment scale. Wherever lab investigations were supposed to be performed to prevent an ADR but was not documented, it was assumed to have been performed. Non-reporting of ADRs may have also affected the observed pattern of results. There is a need for along-term prospective study to evaluate the ADRs due to cancer chemotherapy in India.

\section{CONCLUSION}

Electrolyte disturbance, blood dyscrasias and lung infection were the most commonly reported ADRs in our study. Since $80 \%$ of the ADRs were suspected to be due to platinum containing anticancer drugs this provides a focus for further research on devising methods for prevention or early detection of ADRs. Prompt detection of ADRs is important to decrease morbidity and mortality since those reported in our study were considered non-preventable. Our study also emphasizes the need to encourage the treating physicians to report ADRs which may be considered common and not significant. Considering the increasing number of chemotherapeutic drugs available and their increasing use in the diverse patient population, it is important to track the potential adverse effects of these drugs. There is a need for holistic approach to pharmacovigilance with patients, healthcare professionals, drug manufacturers, drug regulators and policy makers, all having complementary roles to achieve what is envisaged. 
CONFLICT OF INTEREST

None.

The author declare no conflict of interest.

\section{ABBREVIATIONS USED}

ADR: Adverse Drug Reaction; WHO-UMC: World Health Organization-Uppsala Monitoring Centre; SPSS: Statistical Package for the Social Sciences; ESR: Erythrocyte Sedimentation Rate.

\section{ABOUT AUTHOR}

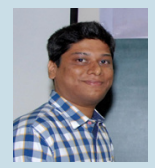

Dr. Sunil Pai B: Is a Postgraduate at the Department of Pharmacology, Kasturba Medical College, Manipal University, Mangalore. He completed his graduation from K.S. Hegde Medical Academy in Mangalore. He has a keen interest in research and is involved in a number of research projects. His subjects of interest include medical oncology, psychopharmacology, drug development and regulatory affairs.

\section{REFERENCES}

1. Lazarou J, Pomeranz BH, Corey PN. Incidence of adverse drug reactions in hospitalized patients: a meta-analysis of prospective studies. JAMA. 1998;279(15):1200-5.

2. Bond CA, Raehl CL. Adverse drug reactions in United States hospitals. Pharmacotherapy. 2006;26(5):601-8.

3. Zhan C, Arispe I, Kelley E, Ding T, Burt CW, Shinogle J. Ambulatory care visits for treating adverse drug effects in the United States, 1995-2001. Jt Comm J Qual Patient Saf. 2005;31(7):372-8.

4. White TJ, Arakelian A, Rho JP. Counting the costs of drug-related adverse events. Pharmacoeconomics. 1999;15(5):445-58.

5. Chabner BA. General Principles of Cancer Chemotherapy. In: Brunton LL, Chabner BA, Knollman BC, editors. Goodman and Gilman's The Pharmacological Basis of Therapeutics. 12 ${ }^{\text {th }}$ ed. New York: Mc Graw Hill Education; 2011. p. 1667-75.

6. Niraula S, Seruga B, Ocana A, Shao T, Goldstein R, Tannock IF. The price we pay for progress: a meta-analysis of harms of newly approved anticancer drugs. $\mathrm{J}$ Clin Oncol. 2012;30(24):3012-9.

7. Safety of Medicines-A Guide to Detecting and Reporting Adverse Drug Reactions-Why Health Professionals Need to Take Action [Internet]. Geneva: World Health Organization; c2002 [cited 2015 Oct 2]. Available from: http://apps.who. int/iris/bitstream/10665/67378/1/WHO_EDM_QSM_2002.2.pdf

8. The use of the WHO-UMC system for standardised case causality assessment [Internet]. Sweden: The Uppsala Monitoring Centre; [cited 2015 Oct 2]. Available from: http://who-umc.org/Graphics/24734.pdf

9. Naranjo CA, Busto U, Sellers EM, Sandor P, Ruiz I, Roberts EA. A method for estimating the probability of adverse drug reactions. Clin Pharmacol Ther. 1981;30(2):239-45

10. Hartwig SC, Siegel J, Schneider PJ. Preventability and severity assessment in reporting adverse drug reactions. Am J Hosp Pharm. 1992;49(9):2229-32.

11. Schumock GT, Thornton JP. Focusing on the preventability of adverse drug reactions. Hosp Pharm. 1992;27(6):538.

12. Wang L. Epidemiology and prevention of adverse drug reactions in the elderly. J Geriatr Cardiol. 2005;2(4):248-53.

13. Merle L, Laroche $M-L$, Dantoine $T$, Charmes J-P. Predicting and preventing adverse drug reactions in the very old. Drugs Aging. 2005;22(5):375-92.

14. Martin RM, Biswas PN, Freemantle SN, Pearce GL, Mann RD. Age and sex distribution of suspected adverse drug reactions to newly marketed drugs in general practice in England: analysis of 48 cohort studies. $\mathrm{Br} \mathrm{J}$ Clin Pharmacol. 1998;46(5):505-11.

15. Surendiran A, Balamurugan N, Gunaseelan K, Akhtar S, Reddy KS, Adithan C. Adverse drug reaction profile of cisplatin-based chemotherapy regimen in a tertiary care hospital in India: An evaluative study. Indian J Pharmacol. 2010;42(1):40-3

16. Mallik S, Palaian S, Ojha P, Mishra P. Pattern of adverse drug reactions due to cancer chemotherapy in a tertiary care teaching hospital in Nepal. Pak J Pharm Sci. 2007;20(3):214-8

17. Poddar S, Sultana R, Sultana R, Akbor MM, Azad MAK, Hasnat A. Pattern of Adverse Drug Reactions Due to Cancer Chemotherapy in Tertiary Care Teaching Hospital in Bangladesh. Dhaka Univ J Pharm Sci. 2009;8(1):11-6.

18. Mallath MK, Taylor DG, Badwe RA, Rath GK, Shanta V, Pramesh CS. The growing burden of cancer in India: epidemiology and social context. Lancet Oncol. 2014; 15(6):205-12.

19. Vassal G, Rubie H, Kalifa C, Hartmann O, Lemerle J. Hyponatremia and renal sodium wasting in patients receiving cisplatinum. Pediatr Hematol Oncol. 1987;4(4):337-44

20. Crawford J, Dale DC, Lyman GH. Chemotherapy-induced neutropenia: risks, consequences, and new directions for its management. Cancer. 2004;100(2):228-37.

21. Mittal N, Gupta MC. Comparison of agreement and rational uses of the WHO and Naranjo adverse event causality assessment tools. J Pharmacol Pharmacother. 2015;6(2):91-3

22. Macedo AF, Marques FB, Ribeiro CF, Teixeira F. Causality assessment of adverse drug reactions: comparison of the results obtained from published decisional algorithms and from the evaluations of an expert panel. Pharmacoepidemiol Drug Saf. 2005;14(12):885-90

23. Belhekar MN, Taur SR, Munshi RP. A study of agreement between the Naranjo algorithm and WHO-UMC criteria for causality assessment of adverse drug reactions. Indian J Pharmacol. 2014;46(1):117-20.

24. Blanc S, Leuenberger $P$, Berger JP, Brooke EM, Schelling JL. Judgments of trained observers on adverse drug reactions. Clin Pharmacol Ther. 1979;25(5):493-8

25. Karch FE, Smith CL, Kerzner B, Mazzullo JM, Weintraub M, Lasagna L. Adverse drug reactions-a matter of opinion. Clin Pharmacol Ther. 1976;19(5 pt 1):489-92. 